\title{
Struttura e contenuti del Rapporto Ambientale
}

\author{
Raffaele Pepe $^{1}$, SALVATORE GIUGLIANO ${ }^{1}$, and Giuseppe Sabatino ${ }^{1}$ \\ ${ }^{1}$ Affiliation not available
}

\begin{abstract}
Il rapporto ambientale sulla valutazione ambientale strategica (VAS), è il documento che individua tutti gli impatti positivi e negativi sul territorio del piano, sviluppando delle possibili alternative di miglioramento, tenendo in considerazione le esigenze del territorio stesso. Questo documento permette di avere una panoramica dedicata ai contenuti principali del piano ed un' analisi dell'opzione ' 0 ", intesa come l'evoluzione del territorio senza l'attuazione del piano. L'analisi vera e propria consiste nell'esaminare le caratteristiche ambientali, culturali e paesaggistiche per poi verificarne gli impatti provocati dal P.U.C. sull'ambiente, con l'obiettivo di mitigarli. È importante non trascurare i processi di monitoraggio sulla mitigazione degli impatti negativi, affinché vengano rispettati gli standard qualitativi e quantitativi degli obiettivi preposti.
\end{abstract}

\section{Introduzione}

Nel Rapporto Ambientale sono “ individuati, descritti e valutati gli effetti significativi che l'attuazione del Piano o del programma potrebbe avere sull'ambiente e sul patrimonio culturale, nonché le ragionevoli alternative che possono adottarsi in considerazione degli obiettivi e dell'ambito territoriale del P/P stesso ". L'elaborazione del rapporto ambientale, una volta individuati e condivisi gli indirizzi generali definiti durante la fase di scoping, si articola in fasi di natura "tecnica" che hanno lo scopo di verificare l'adeguatezza del Piano al contesto programmati- 
co, pianificatorio e fisico di riferimento. Nello specifico il rapporto ambientale è il documento che in primis individua, descrive e valuta gli impatti significativi sulle componenti ambientali, aria, clima acustico, acqua, suolo e sottosuolo, paesaggio, natura, verde urbano e patrimonio storico e culturale e sulla salute derivanti dall'attuazione del piano o del programma; descrive e valuta le ragionevoli alternative, tenendo conto di quanto emerso dalla consultazione dei soggetti competenti in materia ambientale. ${ }^{1}$

Infine il rapporto ambientale concorre alla definizione degli obiettivi e delle strategie del piano indicando i criteri di compatibilità ambientale, gli indicatori ambientali di riferimento e le modalità per il monitoraggio.

Per la redazione del rapporto ambientale sono utilizzate le informazioni pertinenti agli impatti ambientali disponibili nell'ambito di piani o programmi sovraordinati, nonché di altri livelli decisionali. Per facilitare l'informazione e la partecipazione del pubblico, il rapporto ambientale è in genere accompagnato da una sintesi non tecnica che illustra con linguaggio non specialistico i contenuti del rapporto ambientale.

Il procedimento di VAS e la redazione del rapporto ambientale sono regolamentati dal D.Lgs. 152/2006, dalla legge regionale della Campania

\section{Analisi e Sostenibilità}

Il rapporto ambientale è fondamentale per l'analisi di efficienza e sostenibilità di un territorio. Per esaminare al meglio e per approfondire il discorso, si può fare rifermento alla struttura ed ai contenuti del piano urbanistico del comune di San Valentino Torio (SA) ${ }^{2}$, il quale si pone i principali obiettivi di: conservazione dell'identità storica e culturale, salvaguardia delle aree naturali, ed integrazione mediante il potenziamento delle reti commerciali e produttive esistenti. Inoltre vi è un ulteriore punto tra gli obiettivi principali del piano, non di secondaria importanza, bensì ha un 
ruolo fondamentale nella progettazione odierna, ovvero il concetto di sostenibilità ${ }^{3}$, perseguita dal piano tramite un processo di addensamento e di ridisegno dei centri urbani di San Valentino Torio verso forme urbane adeguatamente dotate di verde e di servizi ${ }^{4}$.

Per quanto riguarda lo stato attuale dell'ambiente nel centro urbano di San Valentino Torio sono presenti molti impianti industriali ancora in esercizio che determinano un congestionamento del centro urbano e che pertanto dovrebbero essere delocalizzati in aree congrue a soddisfare anche richieste di eventuali ampliamenti dell' attività stesse ${ }^{5}$. Ad impattare negativamente sul territorio, oltre alle attività industriali, concorre anche il fenomeno dell' abusivismo che ha creato una sorta di "urbanistica parallela", il cui effetto negativo è stato un "consumo di suolo" soprattutto in ambito rurale. Questo fenomeno definito come un'evoluzione spontanea porterebbe al totale impiego dei suoli, oltre che al sostanziale abbandono dell' agricoltura ed alla crescita caotica degli insediamenti lungo le arterie stradali, con conseguente congestione delle attività insediate.

Nella fattispecie si parte da uno studio delle principali caratteristiche ambientali, culturali e paesaggistiche del territorio comunale, che possono essere significativamente interessate dalle trasformazioni previste dal piano, effettuando così un'analisi ambientale finalizzata a costruire un quadro di sintesi delle specificita territoriali, ambientali e socio-economiche, per il Comune di San Valentino Torio, in termini di sensibilita, criticita e opportunita, tramite il quale calibrare obiettivi e azioni del PUC. Si tratta di un'analisi di tipo ricognitivo che consenta di ricostruire un quadro piu aggiornato possibile delle informazioni ambientali disponibili. I dati ambientali e territoriali raccolti e presentati nel Rapporto Ambientale sono stati, quindi, organizzati in rapporto alle seguenti "aree tematiche": popolazione, qualità dell'aria, rumore, inquinamento elettromagnetico, acque superficiali e sotterranee, suolo, produzione e gestione rifiuti, paesaggio e patrimonio storico-culturale, ambiente urbano e rurale. Dopo aver eseguito quest' analisi ricognitiva si illustrano e verificano le modalità secondo le quali il PUC in riferimento alle sue specifiche attribuzioni e competenze, ha fatto propri ed ha perseguito gli obiettivi di protezione ambientale stabiliti a li- 
vello internazionale, comunitario, nazionale e regionale e, piu in generale, in che modo il Piano ha preso in considerazione la questione ambientale nella definizione dei propri obiettivi, delle proprie strategie ed azioni di intervento. Successivamente si procede alla verifica delle interferenze rapportando le visioni strategiche del PUC con gli obiettivi di protezione ambientale individuati precedentemente, attraverso la costruzione della seguente matrice:

Laddove sono state evidenziate interferenze (es. interferenze delle valutazioni strategiche relative alla componente ambientale "popolazione e salute umana") si procede alla valutazione rapportando gli obiettivi del PUC con gli obiettivi di protezione ambientale individuati precedentemente.

Valutata la coerenza delle Visioni Strategiche del PUC con gli obiettivi di protezione ambientale stabiliti a livello internazionale, nazionale, regionale e comunitario, si procede a valutare gli effetti che gli obiettivi producono sulle componenti ambientali. Prendiamo come esempio la visione strategica riguardante la conservazione, tutela e valorizzazione dell'identità ambientale storica, culturale e insediativa.

Quindi si vanno a valutare i possibili impatti significativi sull'ambiente, compresi aspetti quali la biodiversita, la popolazione, la salute umana, la flora e la fauna, il suolo, l'acqua, l'aria, i fattori climatici, i beni materiali, il patrimonio culturale, architettonico e archeologico ed il paesaggio. Per fare ciò viene effettuata una valutazione qualitativa, e successivamente quantitativa. La verifica qualitativa si realizza mediante la costruzione di una matrice che ci permetterà di individuare se gli obiettivi del PUC determinano impatti sulle componenti ambientali (potenzialmente postivi, negativi o nulli).

Successivamente dopo la valutazione qualitativa si esegue la valutazione quantitativa degli impatti, dove per ognuno degli obiettivi previsti dal piano, attraverso opportuni indicatori, vengono quantificati gli effettivi impatti sulle componenti ambientali (stabile negativo o positivo e decremento negativo o positivo). Si vede come gli impatti negativi generati dagli obiettivi di questa visione strategica riguardano diversi aspetti quali popolazione, paesaggio e beni culturali e suolo. 


\section{Conclusioni}

In conclusione vengono adottate le misure di mitigazione e compensazione per ridurre e compensare gli eventuali effetti negativi del piano, indicandone poi le azioni per il miglioramento della sostenibilità ambientale in fase di attuazione, che articola i suoi contenuti progettuali in due componenti, una programmatica ed un'altra strutturale. Infine gli impatti vengono definiti attraverso degli indicatori secondo il modello DPSIR. Al termine dell' analisi, individuati gli impatti negativi, vengono presentate le misure di riduzione e compensazione degli stessi. In particolare gli interventi più interessanti riguardano la popolazione (incentivare la fruizione pubblica dei complessi edilizi storici), il suolo (salvaguardare gli spazi inedificati e i giardini storici), paesaggio e beni culturali (prevedere un piano colore che disciplini gli interventi sulle cortine edilizie) e le acque superficiali (diffondere e favorire un approccio "combinato" nella pianificazione e gestione integrata, su scala di bacino, ai fini della riduzione alla fonte di specifici fattori di inquinamento delle acque determinato dai pesticidi, dai fitofarmaci e dai concimi chimici utilizzati nella pratica agricola $)^{6}$.

\section{References}

1.Naddeo, V., Belgiorno, V., Zarra, T. \& Scannapieco, D. Dynamic and embedded evaluation procedure for strategic environmental assessment. Land Use Policy 31, 605-612 (2013).

2.Piano Urbanistico Comunale di San Valentino Torio (SA), LR 16/2004 - Reg. Regionale 5/2011 del Gennaio 2019..

3.Naddeo, V. \& Korshin, G. Water energy and waste: The great European deal for the environment. 
Science of The Total Environment 764, 142911 (2021).

4.Nesticò, A., Elia, C. \& Naddeo, V. Sustainability of urban regeneration projects: Novel selection model based on analytic network process and zero-one goal programming. Land Use Policy $\mathbf{9 9 ,}$ $104831(2020)$.

5.Zarra, T., Naddeo, V., Giuliani, S. \& Belgiorno, V. Odour Impact Assessment in Industrial Areas. Chemical engineering transaction (2012).

6.Ensano, B. M. B. et al.. Applicability of the electrocoagulation process in treating real municipal wastewater containing pharmaceutical active compounds. Journal of Hazardous Materials 361, 367-373 (2019). 


\section{Figure Captions}

Figure 1. Bosco Verticale (fonte: unsplash.com)

Figure 2. Verifica delle interferenze in relazione alle visioni strategiche dello strumento urbanistico vigente

Figure 3. Valutazione delle interferenze in relazione allo strumento urbanistico vigente

Figure 4. Visione strategica conservazione e tutela patrimonio storico-culturale 


\section{Figures}

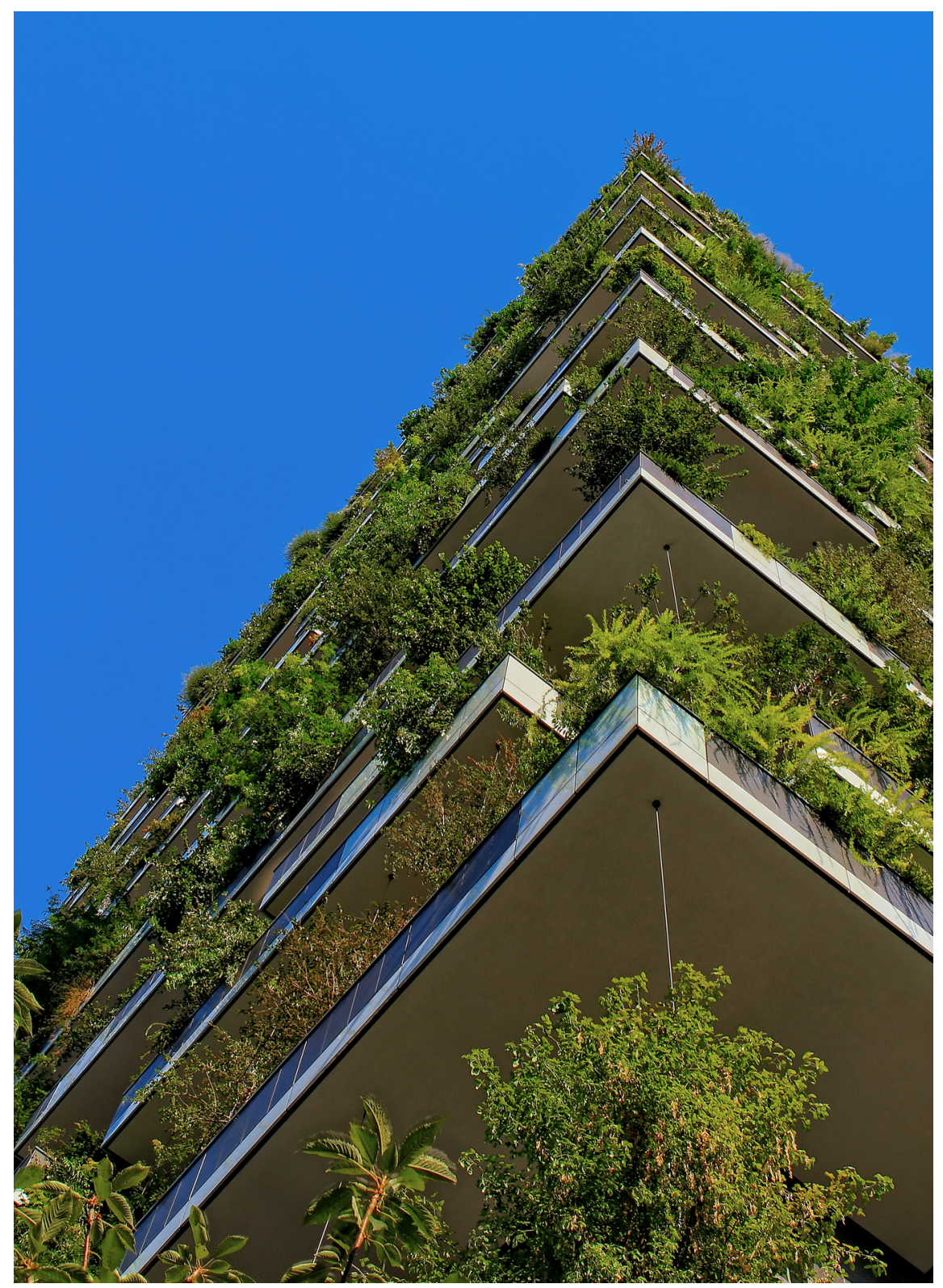

Figure 1: Bosco Verticale (fonte: unsplash.com) 


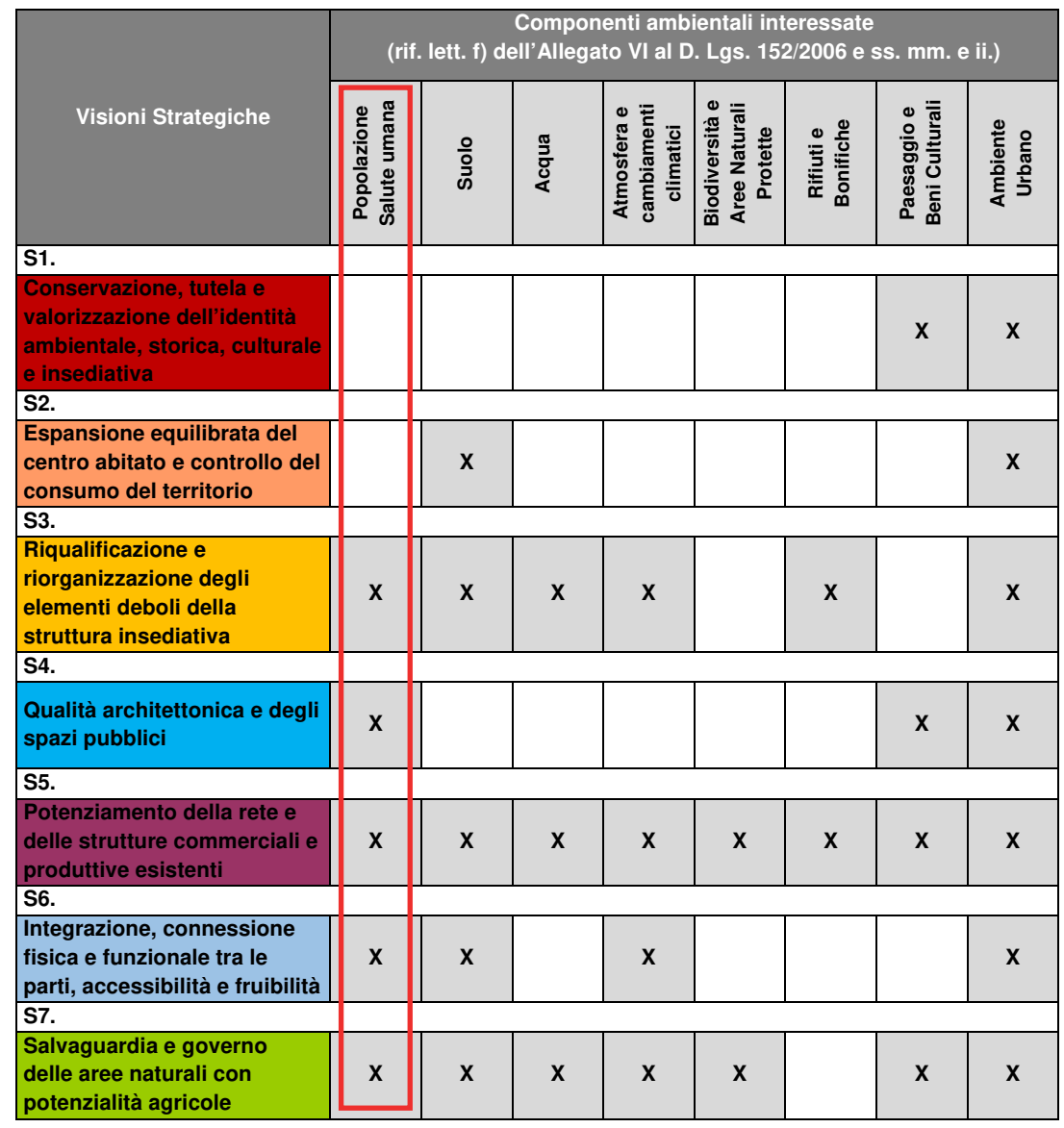

Figure 2: Verifica delle interferenze in relazione alle visioni strategiche dello strumento urbanistico vigente 


\begin{tabular}{|c|c|c|}
\hline Simbolo & Giudizio & Criterio \\
\hline$!$ & Coerente & $\begin{array}{r}\text { L'obiettivo specifico del PUC contribuisce al raggiungimento dell'obiettivo } \\
\text { di protezione ambientale confrontato }\end{array}$ \\
\hline $\mathbf{P}$ & Incoerente & $\begin{array}{r}\text { L'obiettivo specifico del PUC incide negativamente per il raggiungimento } \\
\text { dell'obiettivo di protezione ambientale confrontato }\end{array}$ \\
\hline( & Indifferente & Non si rilevano relazioni, dirette o indirette, fra gli obietti messi a confronto \\
\hline
\end{tabular}

\begin{tabular}{|c|c|c|c|c|}
\hline & & \multicolumn{2}{|c|}{ Popolazione e Salute umana } & \multirow[b]{2}{*}{$\mathrm{Sa} 4$} \\
\hline & Sa1 & Sa2 & Sa3 & \\
\hline $\begin{array}{l}\text { S.3 Riqualificazione e } \\
\text { riorganizzazione degli elementi } \\
\text { deboli della struttura insediativa }\end{array}$ & ( & ( & ( & ( \\
\hline $\begin{array}{l}\text { S4. Qualità architettonica e degli } \\
\text { spazi pubblici }\end{array}$ & $!$ & ( & ( & ( \\
\hline $\begin{array}{l}\text { S.5 Potenziamento della rete e } \\
\text { delle strutture commerciali e } \\
\text { produttive esistenti }\end{array}$ & $\mathrm{P}$ & $\mathrm{P}$ & ( & ( \\
\hline $\begin{array}{l}\text { S. } 6 \text { Integrazione, connessione } \\
\text { fisica e funzionale tra le parti, } \\
\text { accessibilità e fruibilità }\end{array}$ & $\mathrm{P}$ & $\mathrm{P}$ & ( & ( \\
\hline $\begin{array}{l}\text { S.7 Salvaguardia e governo delle } \\
\text { aree naturali con potenzialità } \\
\text { agricole }\end{array}$ & $!$ & ( & ( & ( \\
\hline
\end{tabular}

\begin{tabular}{|c|c|l|}
\hline \multirow{4}{*}{$\begin{array}{c}\text { Popolazione } \\
\text { e Salute }\end{array}$} & Sa1 & Ridurre la percentuale di popolazione esposta agli inquinamenti \\
\cline { 2 - 3 } umana & Sa2 & Ridurre gli impatti delle sostanze chimiche pericolose sulla salute umana e sull'ambiente \\
\cline { 2 - 3 } & Sa3 & Ridurre il grado di accadimento di incidente rilevante nel settore industriale \\
\cline { 2 - 3 } & Sa4 & Migliorare l'organizzazione e la gestione sanitaria \\
\hline
\end{tabular}

Figure 3: Valutazione delle interferenze in relazione allo strumento urbanistico vigente

\begin{tabular}{|c|c|}
\hline VISIONE STRATEGICA & OBIETTIVI \\
\hline \multirow{3}{*}{$\begin{array}{l}\text { S.1 Conservazione, } \\
\text { tutela e valorizzazione } \\
\text { dell'identità ambientale, } \\
\text { storica, culturale e } \\
\text { insediativa }\end{array}$} & $\begin{array}{l}\text { Ob1. Salvaguardia del patrimonio storico espressione della cultura locale e } \\
\text { della memoria collettiva }\end{array}$ \\
\hline & Ob2. Manutenzione dei caratteri storici e documentari \\
\hline & $\begin{array}{l}\text { Ob3. Ricucire il rapporto con il tessuto di recente formazione mediante } \\
\text { l'inserimento di dotazioni territoriali e di servizi }\end{array}$ \\
\hline
\end{tabular}

Figure 4: Visione strategica conservazione e tutela patrimonio storico-culturale 